\title{
Aggregate QTCF Interval
}

National Cancer Institute

\section{Source}

National Cancer Institute. Aggregate QT CF Interval. NCI Thesaurus. Code C117786.

A QT aggregate interval corrected for heart rate using Fridericia's formula. 